\title{
FACTORES ASOCIADOS A LA DISTORSIÓN DE LA IMAGEN CORPORAL EN MUJERES ADOLESCENTES.
}

\author{
FACTORS ASSOCIATED WITH BODY IMAGE DISTORTION IN ADOLESCENT WOMEN.
}

García Villegas Elsy Aidé ${ }^{1}$, Juárez Martínez Liliana ${ }^{1}$, Sámano Reyna ${ }^{2}$, Márquez González Horacio ${ }^{3}$, Martínez Torres Pico Diana Laura ${ }^{4}$, Lamar Rea Violeta Josahandi ${ }^{4}$, Merino Zeferino Beatriz ${ }^{1}$.

1 Instituto Nacional de Ciencias Médicas y Nutrición Salvador Zubirán. 2 Instituto Nacional de Perinatología Isidro Espinosa de los Reyes I. 3 Hospital Infantil de México Federico Gómez. 4 Universidad del Valle de México, Campus Lomas Verdes. México.

Citation: García Villegas E.A., Juárez Martínez L., Sámano R., Márquez González H., Martínez Torres Pico D.L., Lamar Rea V.J., Merino Zeferino B. (2021) Factores asociados a la distorsión de la imagen corporal en mujeres adolescentes. Revista Salud Pública y Nutrición, 20 (1), 12-19.

Editor: Esteban G. Ramos Peña, Dr. CS., Universidad Autónoma de Nuevo León, Facultad de Salud Pública y Nutrición, Monterrey Nuevo León, México. Copyright: (C2021 García Villegas E.A., et al. This is an open-access article distributed under the terms of Creative Commons Attribution License [CC BY 4.0], which permits unrestricted use, distribution, and reproduction in any medium, provided the original author and source are credited.

Competing interests: The authors have declared that no competing interests exist.

DOI: https://doi.org/10.29105/respyn20.1-2

Recibido: 09 de octubre 2020;

Aceptado: 30 de noviembre 2020

Email: meze_us@yahoo.com.mx 


\title{
FACTORES ASOCIADOS A LA DISTORSIÓN DE LA IMAGEN CORPORAL EN MUJERES ADOLESCENTES.
}

\author{
García Villegas Elsy Aidé ${ }^{1}$, Juárez Martínez Liliana ${ }^{1}$, Sámano Reyna², Márquez González Horacio ${ }^{3}$, Martínez \\ Torres Pico Diana Laura ${ }^{4}$, Lamar Rea Violeta Josahandi ${ }^{4}$, Merino Zeferino Beatriz ${ }^{1}$.
}

\begin{abstract}
1 Instituto Nacional de Ciencias Médicas y Nutrición Salvador Zubirán. 2 Instituto Nacional de Perinatología Isidro Espinosa de los Reyes I. 3 Hospital Infantil de México Federico Gómez. 4 Universidad del Valle de México, Campus Lomas Verdes. México.
\end{abstract}

\begin{abstract}
RESUMEN
Introducción. En la adolescencia aumentan las necesidades nutricionales y se adquieren nuevos hábitos alimentarios, debido a influencias psicológicas y sociales que condicionan la aparición de distorsión de la imagen corporal. Objetivo: Identificar la relación de la distorsión de la imagen corporal en mujeres adolescentes con su estado de nutrición, síntomas depresivos, hábitos alimentarios, actividad física y sedentarismo. Material y Método: Estudio transversal en 174 adolescentes en 2018. Se evaluó el estado de nutrición por índice de masa corporal, se auto aplicó un cuestionario para conocer la percepción de imagen corporal, síntomas depresivos, hábitos alimentarios, actividad física y sedentarismo. Se realizó un análisis de regresión logística. La razón de momios se presenta con intervalos de confianza al $95 \%$. Se consideró estadísticamente significativo $p$ $<0.05$. Resultados: La prevalencia de distorsión de imagen corporal fue $63.2 \%, 52.3 \%$ subestimaron su percepción, $50.6 \%$ tenían sobrepeso y obesidad y $43.7 \%$ presentaron síntomas depresivos. Las mujeres con distorsión realizan menos número de comidas, son más jóvenes y presentan mayor prevalencia de sobrepeso y obesidad. Conclusiones: Las adolescentes con distorsión de imagen corporal tienen mayor frecuencia de sobrepeso y obesidad, esto condiciona a mayor riesgo en la salud. Palabras Clave: Distorsión de la imagen corporal, adolescentes, estado de nutrición.
\end{abstract}

\section{ABSTRACT}

Introduction: In adolescence, nutritional needs increase and new eating habits are acquired, due to psychological and social influences that condition the appearance of distortion of the body image. Objective: identify the relationship of the distortion of body image in adolescent women with their nutritional status, depressive symptoms, eating habits, physical activity, and sedentary lifestyle. Material and method: Cross-sectional study in 174 adolescents in 2018. The nutritional status was evaluated by body mass index, a questionnaire was self-applied to know the perception of body image, depressive symptoms, eating habits, physical activity, and sedentary lifestyle. A logistic regression analysis was performed. The odds ratio is presented with $95 \%$ confidence intervals. $\mathrm{P}<0.05$ was considered statistically significant. Results: The prevalence of body image distortion was $63.2 \%, 52.3 \%$ underestimated their perception, $50.6 \%$ were overweight and obese, and $43.7 \%$ presented depressive symptoms. Women with distortion eat fewer meals, are younger, and have a higher prevalence of overweight and obesity. Conclusions: Adolescents with distorted body image are more often overweight and obese, this conditions a higher health risk.

Key words: Distortion of body image, adolescents, nutrition status. 


\section{Introducción}

En la adolescencia aumentan las necesidades nutricionales y se adquieren nuevos hábitos alimentarios debido a influencias psicológicas y sociales que pueden condicionar a una distorsión de la imagen corporal, frecuentemente relacionada con la aparición de trastornos alimentarios (Bobadilla, 2014; Albarello, 2018; Kazarez, 2018), siendo las mujeres las más afectadas por diferentes aspectos socioculturales (Ceja, Vizcaíno, Tapia, Cadenas, Baltazar y Montero, 2010) y además en esta etapa aumenta la preocupación por la imagen corporal (Soto et al; 2015).

La autopercepción de la imagen corporal es la representación simbólica que cada individuo construye sobre su propio cuerpo y esta puede ser correcta, subestimada o sobrestimada. Cuando existe una alteración en la percepción de la imagen y el tamaño corporal no coincide con el real, se habla de que existe una distorsión (Bobadilla 2014; Jiménez, P., Jiménez, A. y Bacardi, 2017). Una mala percepción de la imagen corporal se asocia con una mayor angustia psicológica, más trastornos alimentarios, atracones y menos conductas que promueven la salud, como la actividad física y el consumo de frutas y verduras (Roberts y Duong, 2013).

Se han realizado diferentes estudios para conocer la frecuencia de distorsión en adolescentes, en Túnez fue de $44.8 \%$ (Ben et al; 2019) y en Corea de $51.8 \%$ (Hyun et al; 2014). Heshmat et al. (2015) identificaron el $40 \%$ de distorsión en mujeres adolescentes.

Las causas de la distorsión de la imagen corporal pueden deberse a factores sociales y psicológicos. Entre los sociales se encuentran los medios de comunicación, la cultura, las amistades y la familia (Jiménez, 2017) y dentro de los psicológicos, los síntomas depresivos y la baja autoestima que aumentan la vulnerabilidad a las presiones sobre el cuerpo, influyendo en la distorsión e insatisfacción de la imagen corporal (Hyun, 2014; Quek, 2017; Murray, 2018).

En México, el 35.8\% de las mujeres de 12 a 19 años presentaban sobrepeso u obesidad en 2012 (ENSANUT 2012), incrementando 5.3 puntos porcentuales para la encuesta realizada en el 2018
(41.1 \%) (ENSANUT, 2018), lo que puede representar un riesgo para el desarrollo de distorsión de la imagen corporal. Un estudio realizado en adolescentes mexicanas con sobrepeso y obesidad encontró que el $43.7 \%$ tenían distorsión (Merino, García, Márquez, Guarneros, Sámano y Madrigal, 2018) y (Sámano et al. 2015) señalan que los jóvenes con sobrepeso y obesidad subestiman su peso ya que se ven más delgados de lo que son. Estos autores describen una asociación entre la distorsión de la imagen corporal con el sobrepeso y la obesidad, siendo éstas, un problema relevante en adolescentes, no sólo por sus implicaciones en la salud debido a su relación con las enfermedades crónicas no transmisibles (Durán, 2013), sino también porque afecta el estado mental y la forma en que se relacionan con su entorno.

El objetivo del estudio fue identificar la relación de la distorsión de la imagen corporal en mujeres adolescentes con su estado de nutrición, síntomas depresivos, hábitos alimentarios, actividad física y sedentarismo.

\section{Material y Método}

Estudio transversal realizado en 197 adolescentes de 12 a 18 años de una escuela privada del Estado de México en el 2018. Los criterios de inclusión fueron la participación voluntaria de las alumnas que acudieron a clases el día de la evaluación y que llevaron la autorización firmada por sus padres o tutores. Se obtuvo el consentimiento informado de los responsables de las estudiantes y el asentimiento informado de las alumnas. Se eliminaron del estudio a las alumnas que tenían incompletos los cuestionarios. La investigación fue aprobada por las autoridades escolares.

El procedimiento consistió en la evaluación antropométrica de peso y talla y se realizó un cuestionario auto aplicado para evaluar la autopercepción de la imagen corporal, síntomas depresivos, hábitos alimentarios, actividad física y sedentarismo.

Personal estandarizado por el método de Habitch (1974) efectuó las mediciones antropométricas. Para obtener el peso se utilizó una báscula electrónica de piso marca TANITA UM-061 con una precisión de $100 \mathrm{~g}$, se pesaron con un mínimo de ropa y sin zapatos. La estatura se midió con un estadímetro 
marca SECA 206 con una precisión de $1 \mathrm{~mm}$, de pie, en posición erguida y sin zapatos. Las medidas antropométricas se realizaron bajo las técnicas descritas por Lohman (1991). Con el peso y la estatura se calculó el índice de masa corporal (IMC) dividiendo el peso entre la estatura al cuadrado, el IMC se clasificó acorde con los percentiles de los patrones de referencia de la OMS (2007) para niñas de 5 a 18 años según la edad y sexo para clasificar el estado de nutrición en bajo peso, normal, sobrepeso y obesidad.

La depresión se midió con el Cuestionario de Depresión Infantil (CDI) (Kovacs, 1981) validado en niños y adolescentes para detectar síntomas depresivos (SD). El cuestionario consta de 27 reactivos enunciados en tres frases que rescatan la distinta intensidad o frecuencia de la presencia de síntomas depresivos en adolescentes. La valoración de cada reactivo se hizo utilizando una escala de 0 a 2 puntos, donde $0=$ normalidad (intensidad o frecuencia baja), 1=cierta intensidad (intensidad o frecuencia media) y $2=$ presencia de algún síntoma depresivo (intensidad o frecuencia alta). La puntuación se obtuvo sumando los valores de cada uno de los reactivos. La clasificación fue: $\geq 19$ puntos (presencia de SD claros), 12-18 puntos (problemas psicológicos) y $<12$ puntos (normalidad).

Para evaluar la autopercepción de la imagen corporal, se utilizaron las siluetas de Rand (2000). Se pidió a las participantes elegir una de las 9 siluetas en la que mejor se percibieran y se clasificaron como: 1 2 , bajo peso; 3-5, normal; 6-7, sobrepeso y 8-9 obesidad.

La distorsión de la imagen corporal se obtuvo comparando la silueta seleccionada por cada una de las estudiantes con el estado de nutrición real obtenido mediante el IMC. Los resultados se clasificaron en subestimación, cuando se perciben con un menor estado de nutrición al real, estimación correcta, se perciben igual al estado de nutrición que presentan y sobreestimación, se perciben con un estado nutricio mayor al que tienen (Rizo, Cortés, Brauer, Kuzmar, García, 2014).

Los hábitos alimentarios evaluados fueron si desayuna, tiempos de comida al día, consumo de refresco y agua. Se preguntó si realizaban actividad física y para conocer el sedentarismo se preguntó el tiempo frente a la pantalla de televisión, de aparatos electrónicos o video juegos.

Análisis estadístico: Las variables continúas se presentaron como mediana y rango intercuartílico (RIQ) y fueron comparadas mediante la prueba $\mathrm{U}$ de Mann-Whitney. Las variables cualitativas se describieron mediante frecuencias $y$ fueron comparadas entre grupos utilizando la prueba de X2 de Pearson Se realizó análisis de regresión logística siendo la variable dependiente la distorsión de la imagen corporal. El coeficiente de regresión (beta) o razón de momios (OR) se presenta con sus intervalos de confianza al $95 \%$ (IC $95 \%$ ). Los diagnósticos de regresión se realizaron mediante la prueba de Hosmer-Lemeshow para el modelo logístico. Se consideró estadísticamente significativo $\mathrm{p}<0.05$. El análisis se realizó con el paquete estadístico SPSS versión 21.0 para Windows (IBM Corp, 12).

\section{Resultados}

De un total de 197 adolescentes, se tuvo una tasa de respuesta de $88.3 \%$, obteniendo información de 174 participantes de 12 a 18 años. La prevalencia de distorsión de la imagen corporal fue del $63.2 \%$, el $52.3 \%$ subestimaron su percepción, el $50.6 \%$ tenían sobrepeso y obesidad y el $43.7 \%$ presentaron síntomas depresivos claros (tabla 1).

\begin{tabular}{|c|c|}
\hline Variables & $\begin{array}{c}\text { Población } \\
(n=174) \\
\text { Mediana }\left(\mathrm{RIQ}^{*}\right)\end{array}$ \\
\hline$\overline{\text { Edad (años) }}$ & $14.0(13.1-15.0)$ \\
\hline Peso (kg) & $56.5(50.2-62.4)$ \\
\hline Estatura $(\mathrm{cm})$ & $157(153.3-161.2)$ \\
\hline IMC (kg/m2) & $22.6(20.4-25.3)$ \\
\hline Síntomas depresivos (puntaje) & $11(7.0-16.2)$ \\
\hline Estado de nutrición (IMC) & n (\%) \\
\hline Bajo peso & $28(16.1)$ \\
\hline Normal & $58(33.3)$ \\
\hline Sobrepeso & $60(34.5)$ \\
\hline Obesidad & $28(16.1)$ \\
\hline Síntomas depresivos claros & $76(43.7)$ \\
\hline Distorsión de la imagen corporal & $110(63.2)$ \\
\hline Subestimación & $91(52.3)$ \\
\hline Sobreestimación & 19 (10.9) \\
\hline
\end{tabular}


En la tabla 2 se observa que el comportamiento de los síntomas depresivos fue similar en las que presentaron y no distorsión. Con respecto a los hábitos alimentarios, 4 de cada 10 alumnas no desayunaban, realizaban una o dos comidas y bebían menos de un litro de agua al día. La mitad de las adolescentes no practicaba actividad física y $36.2 \%$ pasaba más de 7 horas al día frente a una pantalla, además se observó una asociación entre el consumo de 1 a 2 comidas al día y la distorsión de la imagen corporal.

\begin{tabular}{|c|c|c|c|}
\hline Variables & $\begin{array}{c}\text { Población } \\
\text { total } \\
(n=174)\end{array}$ & $\begin{array}{c}\text { Distorsión } \\
(\mathrm{n}=110)\end{array}$ & $\begin{array}{c}\text { Sin } \\
\text { distorsión } \\
(\mathrm{n}=64)\end{array}$ \\
\hline Síntomas depresivos & $76(43.7)$ & $48(43.6)$ & $28(43.8)$ \\
\hline \multicolumn{4}{|l|}{ Hábitos alimentarios } \\
\hline No desayunar & $69(39.7)$ & $46(41.8)$ & $23(35.9)$ \\
\hline 1-2 comidas al día* & $72(41.4)$ & $54(49.1)$ & $18(28.1)$ \\
\hline Consumo de agua/día < 1 litro & $72(41.4)$ & $48(43.6)$ & $24(37.5)$ \\
\hline Consumo de refresco más de 2 veces/día & $60(34.5)$ & $38(34.5)$ & $22(34.4)$ \\
\hline \multicolumn{4}{|l|}{ Actividad física } \\
\hline No realizan & $84(48.3)$ & $53(48.2)$ & $31(48.4)$ \\
\hline \multicolumn{4}{|l|}{ Sedentarismo } \\
\hline Tiempo frente a pantalla más de 7 horas/día & $63(36.2)$ & $37(33.6)$ & $26(40.6)$ \\
\hline
\end{tabular}

En relación con el estado de nutrición y tener distorsión de la imagen corporal, se encontró una diferencia significativa en la edad de las adolescentes con bajo peso o estado de nutrición normal, en donde las que no presentaron distorsión tenían mayor edad, además aquellas con sobrepeso y obesidad sin distorsión muestran mayor frecuencia de síntomas depresivos (tabla 3 ).

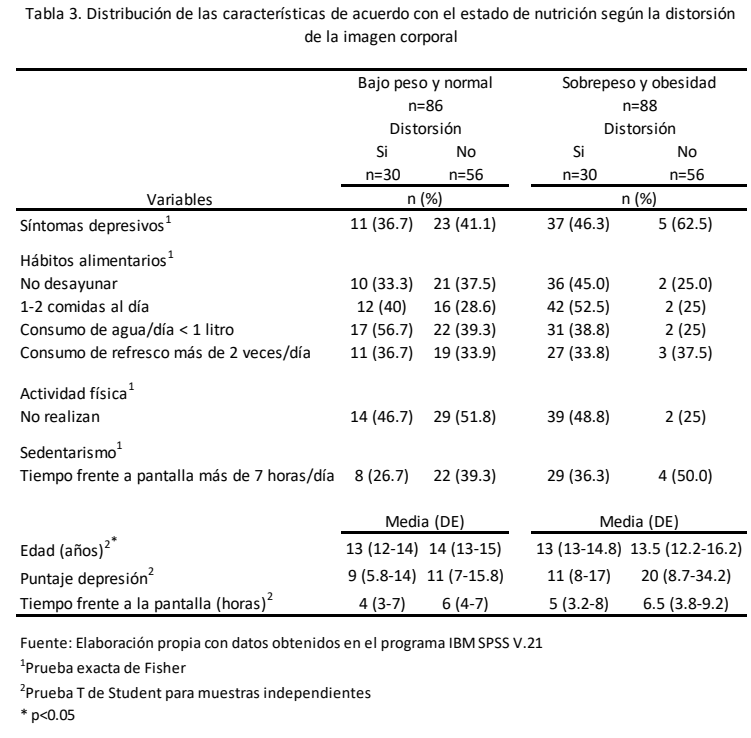

En la tabla 4 se muestra que la presencia de distorsión de la imagen corporal fue mayor en las adolescentes con sobrepeso y obesidad, y además se observa que la mayor proporción de ellas subestiman su estado de nutrición.

Tabla 4. Asociación del estado de nutrición con la distorsión de la imagen corporal

\begin{tabular}{lccc}
\hline \multirow{2}{*}{$\begin{array}{c}\text { Distorsión de la imagen } \\
\text { corporal }\end{array}$} & \multicolumn{3}{c}{ Estado de nutrición } \\
\cline { 2 - 3 } & $\begin{array}{c}\text { Bajo peso y } \\
\text { normal }(\mathrm{n}=86)\end{array}$ & $\begin{array}{c}\text { Sobrepeso } \\
(\mathrm{n}=69)\end{array}$ & $\begin{array}{c}\text { Obesidad } \\
(\mathrm{n}=28)\end{array}$ \\
\cline { 1 - 2 } $\begin{array}{l}\text { Presencia de distorción* } \\
\text { No }\end{array}$ & $56(65.1)$ & $5(8.3)$ & $3(10.7)$ \\
Si & $30(34.9)$ & $55(91.7)$ & $25(89.3)$ \\
$\begin{array}{l}\text { Estimación de Distorción* } \\
\text { Subestimación }\end{array}$ & $15(17.4)$ & $51(85.0)$ & $25(89.3)$ \\
Sobreestimación & $15(17.4)$ & $4(6.7)$ & $0(0.0)$ \\
\hline $\begin{array}{l}\text { Fuente: Elaboración propia con datos obtenidos en el programa IBM SPSS V.21 } \\
\text { * Chi-cuadrada p<0.05 }\end{array}$ & & &
\end{tabular}

En la tabla 5 se observa que las adolescentes con sobrepeso y obesidad tienen 20.4 veces el riesgo de presentar distorsión de la imagen corporal, y la edad es un efecto adverso, ya que las adolescentes más jóvenes tienen mayor probabilidad de tener distorsión. El 45 \% de la variabilidad en la distorsión corporal se explica por el estado de nutrición y la edad de las adolescentes. Al obtener una prueba de Hosmer y Lemeshow se puede concluir que los datos se ajustan al modelo que estamos postulando con respecto a la variable dependiente. 
Tabla 5. Análisis de regresión logística para variables asociadas a distorsión corporal de las adolescentes

\begin{tabular}{ccccccc}
\hline Variable & Beta & OR & IC 95\% & p & $\begin{array}{c}\text { Prueba de } \\
\text { Hosmery } \\
\text { Lemeshow }\end{array}$ & $\begin{array}{c}\mathrm{R}^{2} \text { de } \\
\text { Ngelkerke }\end{array}$ \\
\hline $\begin{array}{c}\text { Estado de nutrición (bajo peso } \\
\text { y normal/sobrepeso y } \\
\text { Edad (años) }\end{array}$ & 3.02 & 20.4 & $8.4-49.5$ & $<0.001$ & 0.216 & 0.452 \\
\hline
\end{tabular}

Fuente: Elaboración propia con datos obtenidos en el programa IBM SPSS V.21

OR: Odds ratio;IC 95\%: intervalo de confianza del 95\%

\section{Discusión}

En este estudio se evidencia una alta proporción de adolescentes con distorsión de la imagen corporal, más que en otras investigaciones, además tienen mayor prevalencia de sobrepeso y obesidad. En adolescentes coreanas (Hyun et al, 2014) reportan que la distorsión de la imagen corporal se asoció con sobrepeso u obesidad. Mendonça et al. (2014) encontraron que las adolescentes con distorsión de la imagen corporal se perciben con sobrepeso o con peso normal cuando en realidad eran obesas.

Las adolescentes con sobrepeso y obesidad que no tenían distorsión mostraron más síntomas depresivos, a este respecto Roberts y Duong (2013) mencionan que un grupo de jóvenes con sobrepeso u obesidad tienen mayor probabilidad de sufrir depresión y ésta se relaciona con su percepción, estrés y estilos de vida poco saludables que con el tiempo los lleva a desarrollar obesidad.

Se debe destacar que las jóvenes estudiadas con sobrepeso y obesidad subestimaron su condición, situación que se hace particularmente relevante cuando esta prevalencia es mayor incluso que a nivel nacional; lo que concuerda con otros autores (Oliva, Ordóñez, Santana, Marín, Andueza, Gómez, 2016) quienes describen que las adolescentes que subestimaron su peso corporal presentaron obesidad. En este sentido, es importante identificar cómo se perciben dado que éste puede ser el primer impedimento para atenderse o iniciar una estrategia de prevención. Sámano et al (2015) mencionan que las mujeres que tienden a sobreestimar el peso corporal están más comprometidas para buscar ayuda y seguir las recomendaciones de un especialista, contrario a lo que podría pasar con las que subestiman su condición.

Otro estudio encontró que niños escolares con sobrepeso y obesidad, con una prevalencia de $38 \%$ subestiman su peso y seleccionan imágenes ideales más delgadas de las que les corresponden (Uribe et al, 2018). Aun cuando no es el mismo grupo de población que en el presente estudio, da cuenta de que el problema de sobrepeso y obesidad suele no ser percibido por quien lo presenta. Lee J. y Lee, Y. (2016) informaron que aproximadamente uno de cada dos adolescentes de 12 a 18 años percibió incorrectamente su estado de peso corporal, ya sea subestimando $(23.7 \%)$ o sobreestimando $(25.6 \%)$ y que debido a los efectos negativos en la salud física y mental es importante evaluar la prevalencia de distorsión de la imagen corporal.

En nuestro estudio, las adolescentes de menor edad tuvieron una mayor probabilidad de presentar distorsión de la imagen corporal, lo que se puede explicar por lo publicado por Gómez, Sánchez y Mahedero (2013) quienes reportaron que, entre adolescentes de 12 a 17 años de ambos sexos, a medida que aumenta la edad tienen una mejor percepción de su imagen corporal.

La distorsión de la imagen corporal en las adolescentes es preocupante debido a que está influenciado por los modelos estéticos corporales que las lleva a restringir su alimentación o modificar sus hábitos alimentarios (Vaquero, Alacid, Muyor y López, 2013). En este estudio encontramos una diferencia entre la restricción en el número de comidas al día, siendo mayor la frecuencia en las que presentan distorsión. Oliva et al. (2016) refieren que la modificación de los hábitos alimentarios, el realizar dietas y actividad física en busca de conseguir una imagen adecuada a la estética dominante, es un problema de salud emergente en este grupo de edad. Lo anterior puede estar relacionado con casos de trastornos de la conducta alimentaria, como contraparte del sobrepeso y la obesidad. En adolescentes japonesas la percepción de su imagen corporal impacta en sus hábitos alimentarios y en ocasiones buscan suprimir su aporte calórico para lograr modificaciones en su composición corporal (Shirasawa et al, 2015).

La fortaleza de nuestro estudio radica que en este grupo de población se encontró alta prevalencia de distorsión de la imagen corporal, principalmente que subestiman su condición y además presentaron una alta frecuencia de sobrepeso y obesidad, lo que las coloca como un grupo de riesgo y de priorización, en donde se hace necesario la detección de la distorsión y un abordaje de atención integral que considere no 
sólo el elemento nutricional sino también el psicológico. Dentro de las debilidades es que al ser un estudio transversal no es posible establecer una relación causal de la distorsión. Una segunda es que el tamaño de la población estudiada fue pequeño.

\section{Conclusiones}

Los factores asociados a la distorsión de la imagen corporal en el estudio son consumir menos de 2 comidas al día, tener menos edad y presentar sobrepeso y obesidad.

Las mujeres adolescentes con distorsión de la imagen corporal son las que tienen una mayor prevalencia de sobrepeso y obesidad, además subestiman su condición y son las de menor edad. La autopercepción errónea del estado de nutrición ya sea bajo peso, sobrepeso u obesidad, especialmente en este grupo de edad, ha sido una preocupación nutricional para los profesionales de la salud por las consecuencias que conlleva con respecto a su control de peso y hábitos poco saludables. Por lo que se debe considerar cómo se perciben y cómo desean estar, e incorporar en la terapia de apoyo no sólo la parte nutricional sino la detección de eventos como la distorsión, la depresión y el estado psicológico y emocional, ya que estos pueden influir en el manejo efectivo del sobrepeso y la obesidad.

\section{Bibliografía}

Albarello, F., Crocetti, E., y Rubini, M. (2018). I and Us: A Longitudinal Study on the Interplay of Personal and Social Identity in Adolescence. Journal of Youth and Adolescence, 47(4), 689-702. https://doi.org/10.1007/s10964-017-0791-4.

Ben Ayed, H., Yaich, S., Ben Jemaa, M., Ben Hmida, M., Trigui, M., Jedidi, J., Sboui, I., Karray, R., Feki, H., Mejdoub, Y., Kassis, M., \& Damak, J. et al. (2019). What are the correlates of body image distortion and dissatisfaction among school-adolescents? International journal of adolescent medicine and health, /j/ijamh.aheadof-print/ijamh-2018-0279/ijamh-2018-0279.xml. Advance online publication. https://doi.org/10.1515/ijamh-2018-0279

Bobadilla, S., López, A. (2014). Distorsión de imagen corporal percibida e imaginada. Un posible factor para la obesidad y el sobrepeso en mexicanos. Revista Médica del Instituto Mexicano del Seguro Social, 52(4), 408-414.

Ceja, G., Vizcaíno, J., Tapia, R., Cadenas, JL., Baltazar, LM., y Montero, SA. (2010). Depresión en pacientes de la Unidad de Medicina Familiar 19 con diagnóstico de sobrepeso y obesidad. Revista Cubana de Investigaciones Biomédicas, 29(2), 237-243.

Descifr3mo5. (s. f.). Recuperado 5 de octubre de 2020, de https://www.gob.mx/cms/uploads/attachment/fil e/280081/descifremos15-2.pdf.

Durán, S., Rodríguez, M., Record, J., Barra R., Olivares, R., Tapia, A., Moraga, A., Campos, C., Mattar, P., Pérez, G. y Neira, A. (2013). Autopercepción de la imagen corporal en estudiantes universitarios de Chile y Panamá. Revista Chilena de Nutrición, 40(1), 2632. https://dx.doi.org/10.4067/S071775182013000100004 .

Encuesta Nacional de Salud y Nutrición (2012). Resultados Nacionales 2012. Retrieved November 27, 2020, from https://ensanut.insp.mx/encuestas/ensanut2012/d octos/informes.

Encuesta Nacional de Salud y Nutrición. (2018). Informe de Resultados de la Encuesta Nacional de Salud y Nutrición 2018. Retrieved October 5, 2020, from https://ensanut.insp.mx/encuestas/ensanut2018/i nformes.php\%0A.

Gómez, A., Sánchez, B. y Mahedero, M (2013). Insatisfacción y distorsión de la imagen corporal en adolescentes de doce a diecisiete años de edad. Ágora para la educación física y el deporte, 15(1), 54-63.

Habitch, J. (1974). Estandarización de métodos epidemiológicos cuantitativos sobre el terreno. Boletín de la Oficina Sanitaria Panamericana, 375-385.

Heshmat, R., Kelishadi, R., Motamed, N., Motlagh, M. E., Ardalan, G., Arifirad, T., Rastad, H., 
Asayesh, H., Djalalinia, S., Larijani, B., y Qorbani, M. (2015). Association between body mass index and perceived weight status with selfrated health and life satisfaction in Iranian children and adolescents: the CASPIAN-III study. Quality of life research: an international journal of quality of life aspects of treatment, care and rehabilitation, 24(1), 263-272. https://doi.org/10.1007/s11136-014-0757-x.

Hyun, M. Y., Jung, Y. E., Kim, M. D., Kwak, Y. S., Hong, S. C., Bahk, W. M., Yoon, B. H., Yoon, H. W., y Yoo, B. (2014). Factors associated with body image distortion in Korean adolescents. Neuropsychiatric disease and treatment, 10 , 797-802. https://doi.org/10.2147/NDT.S63143.

IBM Corp. Released 2012. IBM SPSS Statistics for Windows, Version 21.0. Armonk, NY: IBM Corp.

Jiménez, P., Jiménez, A., y Bacardi, M. (2017). Body-image dissatisfaction in children and adolescents: a systematic review. Nutrición Hospitalaria, 34(2), 479-489. https://doi.org/10.20960/nh.455.

Kazarez, M., Vaquero R., y Esparza, F. (2018). Percepción y distorsión de la imagen corporal en bailarinas españolas en función del curso académico y de la edad. Nutrición Hospitalaria, 35(3), 661 668. https://dx.doi.org/10.20960/nh.1388.

Kovacs, M., Del Barrio, V., Carrasco, M. Á. (2011). CDI: Inventario de depresión infantil. Madrid, España: TEA.

Lee, J., y Lee, Y. (2016). The association of body image distortion with weight control behaviors, diet behaviors, physical activity, sadness, and suicidal ideation among Korean high school students: a cross-sectional study. BMC Public Health, 16, 39. https://doi.org/10.1186/s12889016-2703-z.

Lohman T, Roche A, Martorell R, (1991) Measurement Descriptions and Techniques; Circumferences. In: Lohman $\mathrm{T}$, Roche A, Martorell R, editores Anthropometric
Standardization Reference Manual. Champaign, IL:Human Kinetics Books.

Mendonça, K., Sousa, A., Carneiro, C., Nascente, F., Póvoa, T., Souza, W., Jardim, T. y Jardim, P. (2014). Does nutritional status interfere with adolescents' body image perception? Eating Behaviors, 15(3), 509-512. https://doi.org/10.1016/j.eatbeh.2014.06.011.

Merino, B., García, E., Márquez, H., Guarneros, N., Sámano, R., y Madrigal, H. (2018). Asociación de síntomas depresivos por tamizaje con el estado nutricional y autopercepción de la imagen corporal en un grupo de adolescentes del estado de México. Revista Mexicana de Endocrinología, Metabolismo y Nutrición; 5(1), 5-12.

Murray, K., Rieger, E., y Byrne, D. (2018). Body image predictors of depressive symptoms in adolescence. Journal of Adolescence, 69, 130139.https://doi.org/10.1016/j.adolescence.2018.1 $\underline{0.002}$.

Oliva, Y., Ordóñez, M., Santana, A., Marín, A., Andueza, G., y Gómez, I. (2016). Concordancia del IMC y la percepción de la imagen corporal en adolescentes de una localidad suburbana de Yucatán. Revista Biomédica, 27(2), 49-60. https://doi.org/10.32776/revbiomed.v27i2.24

Quek, Y. H., Tam, W., Zhang, M., y Ho, R. (2017). Exploring the association between childhood and adolescent obesity and depression: a metaanalysis. Obesity reviews: an official journal of the International Association for the Study of Obesity, 18(7), 742-754. https://doi.org/10.1111/obr.12535.

Rand, C. S. y Resnick, J. L. (2000). The "good enough" body size as judged by people of varying age and weight. Obesity research, 8(4), 309-316. https://doi.org/10.1038/oby.2000.37

Rizo, M., Cortés, E., Brauer, N., Kuzmar, I., y García, M. (2014). Distortion of self-image: Risk factor for obesity in children and teenagers. Revista Española de Nutrición Humana y Dietética. $18.212-217$. http://dx.doi.org/10.14306/renhyd.18.4.90 
Roberts, R. E., y Duong, H. T. (2013). Perceived weight, not obesity, increases risk for major depression among adolescents. Journal of psychiatric research,47(8), 1110-1117. https://doi.org/10.1016/j.jpsychires.2013.03.019.

Sámano, R., Rodríguez, A., Sánchez, B., Godínez, E., Noriega, A., Zelonka, R., Garza, M. y Nieto, J. (2015). Satisfacción de la imagen corporal en adolescentes y adultos mexicanos y su relación con la autopercepción corporal y el índice de masa corporal real. Nutrición Hospitalaria, 31(3), 10821088. https://dx.doi.org/10.3305/nh.2015.31.3.8 $\underline{364}$

Shirasawa, T., Ochiai, H., Nanri, H., Nishimura, R., Ohtsu, T., Hoshino, H., Tajima, N., y Kokaze, A. (2015). The relationship between distorted body image and lifestyle among Japanese adolescents: a population-based study. Archives of public health, 73(1),

32. https://doi.org/10.1186/s13690-015-0082-z

Soto, Mª N., Marín, B., Aguinaga, I., Guillén, F., Serrano, I., Canga, N., Hermoso de Mendoza, J., Stock, C., Kraemer, A. y Annan, J. (2015). Análisis de la percepción de la imagen corporal que tienen los estudiantes universitarios de Navarra. Nutrición Hospitalaria, 31(5), 22692275.

https://dx.doi.org/10.3305/nh.2015.31.5.7418

Uribe, F., Portillo, M., Parra, J., Hernández, S., Alonso, R., Acevedo, M. y Felipe, J. (2018). Autopercepción del peso a través de figuras corporales en niños en edad escolar de Piedras Negras, Coahuila, México. Boletín Médico del Hospital Infantil de México,75(6), 366372. https://doi.org/10.24875/bmhim.18000041

Vaquero, R., Alacid, F., Muyor, J., y López, P. (2013). Imagen corporal: revisión bibliográfica. Nutrición Hospitalaria, 28(1), $27-$ 35. https://dx.doi.org/10.3305/nh.2013.28.1.601

6. 